\title{
Stratégies analytiques en toxicologie d'urgence
}

\section{Analytical strategy in emergency toxicology}

\section{Bernard CAPOLAGHI*(1), Mustapha MOULSMA ${ }^{(2)}$, Nicole HOUDRET ${ }^{(3)}$, Frédéric J. BAUD ${ }^{(4)}$}

(1) Laboratoire de Biochimie-Toxicologie, Hôpital Bel-Air, C.H.R. Metz-Thionville - 57100 THIONVILLE

(2) Laboratoire de Pharmacotoxicologie et Analyse de Traces, Hôpital Édouard Herriot - 69437 LYON Cedex

(3) Laboratoire de Biochimie et de Biologie Moléculaire, Hôpital Calmette, C.H.R.U. - 59037 LILLE Cedex (4) Service de Réanimation Médicale et Toxicologique, Hôpital Lariboisière - 75475 PARIS Cedex 10

*Auteur à qui adresser la correspondance : Bernard CAPOLAGHI, Laboratoire de Biochimie-Toxicologie, Hôpital Bel-Air, C.H.R. Metz-Thionville, rue du Friscaty - 57100 THIONVILLE Tél : 0382558199 - Fax : 0382558201 - e-mail : bcapo@ wanadoo.fr

(Reçu le 3 octobre 2000 ; accepté le 23 octobre 2000)

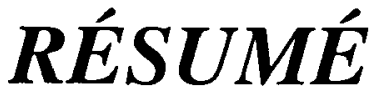

La Toxicologie d'urgence associe la notion d'exploration d'un très vaste domaine de substances chimiques à la notion d'efficacité de prise en charge thérapeutique du sujet intoxiqué. En conséquence, la stratégie analytique à adopter sera le fruit d'une collaboration clinico-biologique étroite comprenant l'approche clinique (anamnèse, signes cliniques), l'approche biologique (gazométrie, osmolalité, ionogramme, ...) et la connaissance des limites et des intérêts des différentes méthodes disponibles localement. Les méthodes spectrophotométriques et immunologiques sont des méthodes de dépistage au champ d'application limité et dont l'intérêt est d'apporter rapidement une orientation sur l'origine de l'intoxication (pesticides, médicaments, substances illicites, ...). Les méthodes séparatives associées à des outils de détection (spectres $U V$, spectres de masse) sont le complément indispensable à l'identification des molécules responsables de l'intoxication. En dernière étape, l'analyse quantitative du produit toxique identifié peut faire appel à une méthode immunologique (paracétamol, digoxine, ...) ou chromatographique (méprobamate, colchicine,...).

MOTS-CLÉS

Toxicologie d'urgence, Intoxication aiguë.

\section{SUMMARY}

Emergency Toxicology involves the screening of a large domain of toxicological products and, at the same time, to give a quick response to assume the best therapy for patients hospitalized in intensive care unit. To fulfill this challenge, analytical strategy shall result from a straight collaboration between clinicians and analysts including clinical and biological signs and the knowing of interest and pitfalls of each laboratory method. Spectrophotometrical and immunological assays are quick screening methods of a limited number of molecules (pesticides, drugs of abuse). Separative methods coupled to specific and sensitive detection (UV or mass spectrometry) shall complete the screening of numerous other toxic substances. Finally, the last step of analysis may be a quantitative measurement of the implied toxic substance(s) involving either immunological assay (acetaminophen, digoxine,..) or chromatographic assay (meprobamate, colchicine,...).

\section{KEY-WORDS}

Emergency toxicology, Acute poisoning. 
Face au grand nombre d'intoxications aiguës (1), tant accidentelles que volontaires, admises en situation d'urgence, il est important de situer l'approche biologique et toxicologique dans le diagnostic, le pronostic et le traitement de ces admissions $(2,3,4,5,6)$. Cette démarche multidisciplinaire est avant tout clinique. Elle nécessite cependant de bonnes relations entre biologistes et cliniciens pour une prise en compte optimale de l'urgence toxicologique et la mise en œuvre d'une stratégie globale spécifique à chaque établissement.

\section{Le diagnostic toxicologique}

Les circonstances de découverte d'un patient présumé intoxiqué constituent la première étape du diagnostic et illustrent l'importance du contexte et des priorités cliniques. Trois cas majeurs peuvent être identifiés :

- Le patient a ingéré ou a été exposé à un toxique défini et l'examen initial est normal.

- Le patient a ingéré ou a été exposé à un toxique défini et l'examen initial révèle des symptômes.

- Le patient présente des symptômes et une origine toxique est suspectée sans toxique défini.

Selon le cas, il s'agira donc soit de confirmer l'intoxication présumée de nature connue ou inconnue et d'en évaluer la gravité, soit d'exclure l'hypothèse d'une intoxication.

A ce stade, le risque ne doit pas être sous-estimé. En effet, la mortalité est 4 fois plus grande chez des patients intoxiqués ne présentant pas de coma et témoigne de la gravité des intoxications à certaines substances comme le paracétamol, la colchicine, les glycols, les inhibiteurs calciques ou les $\beta$ bloquants.

Les études épidémiologiques montrent par ailleurs une forte augmentation du nombre de sujets ingérant plusieurs médicaments simultanément (fuite médicamenteuse) au regard des intoxications à molécule unique (suicide vrai).

L'approche clinique incluant l'anamnèse, l'examen clinique et l'ECG complète cette prise en charge. Il s'agit tout d'abord d'établir la liste des toxiques présumés et de vérifier la compatibilité des symptômes présentés avec la nature de ces substances toxiques. De même, l'adéquation entre l'apparition des signes cliniques et le délai d'ingestion doit être examinée soigneusement.

Les résultats biologiques de base (ionogramme, osmolarité, gazométrie, coagulation...) complètent cette première étape clinique avec éventuellement la fourniture d'analyses biochimiques spécifiques de la toxicité du produit lorsque ce dernier peut être considéré comme toxique lésionnel potentiel (7). En effet, chaque fois qu'une substance toxique perturbe le milieu intérieur, l'analyse biologique prime sur la recherche de l'identité du toxique. A titre d'exemple, la glycémie est plus importante à connaître que la concentration en éthanol lors d'une intoxication alcoolique, de même pour le $\mathrm{pH}$ par rapport à l'éthylène glycol chez un patient ayant ingéré une solution de glycols. L'évaluation de la fonction des organes impliqués dans le métabolisme et l'excrétion des toxiques est donc primordiale ainsi que l'utilisation de critères indirects de toxicité comme le taux de prothrombine pour le suivi des antivitamines $\mathrm{K}$ ou la cholinestérase pour les organophosphorés.

Ces premières étapes prioritaires seront suivies par une approche plus toxicologique qui permettra de caractériser et/ou de doser le toxique ingéré.

Auparavant, on aura pris soin de collecter un maximum d'informations pour tenter d'expliquer les symptômes et orienter les recherches. Un coma calme doit évoquer une intoxication par les benzodiazépines, les carbamates ou les phénothiazines. De même l'appréhension d'un certain nombre de situations cliniques à orientation toxicologique peut bénéficier d'un arbre de décision (8). La recherche du toxique responsable de l'intoxication comprend, bien évidemment, la réalisation de prélèvements biologiques. Il est judicieux de prélever dès l'admission une quantité suffisante d'échantillons pour la constitution d'une sérothèque ou urothèque qui pourra être utilisé a postériori pour l'identification exacte du produit ou une compréhension plus fine de l'intoxication. La recherche toxicologique est un instrument diagnostic utile et parfois indispensable pour l'évaluation de l'état du patient. Or, tous les toxiques ont un impact très différent en termes de facteurs pronostiques ou de critères de gravité (9). Deux grandes catégories peuvent être isolées : les substances toxiques dont la concentration est corrélée de façon contemporaine avec la gravité de l'intoxication (Ethanol, phénobarbital, ...) et les toxiques lésionnels pour lesquels l'utilisation de la concentration en toxique se limite à un facteur pronostic en décalage avec l'état clinique du patient (paracétamol, paraquat, phalloïdine, ...)

\section{Approche biologique et analytique en toxicologie d'urgence}

\section{Collaboration clinico-biologique}

Le diagnostic toxicologique montre la nécessité d'un dialogue entre analyste et clinicien. Il doit s'accompagner d'une demande d'analyses toxicologiques clairement formulée précisant les toxiques suspectés, la natu- 
re des liquides biologiques (sang, urines, liquide gastrique, ....) auxquels ces analyses sont applicables et leurs délais de réalisation.

Ce formulaire correctement rempli doit fournir tous les renseignements nécessaires à l'orientation de la conduite analytique :

- l'état clinique du patient

- les circonstances de l'intoxication (lieu, moment de l'exposition)

- l'heure supposée d'ingestion

- la découverte ou non par les premiers intervenants d'informations telles que les médications habituelles, la présence d'emballages à proximité, ....

- l'heure des prélèvements

- les traitements mis en œuvre avant le prélèvement.

A partir de ces renseignements, la mission du laboratoire d'urgence sera, parallèlement à la réalisation des analyses biologiques de base (ionogramme, osmolalité, gazométrie, ... ) de confirmer une intoxication présumée de nature connue ou inconnue, d'en évaluer la sévérité ou d'exclure une intoxication possible.

Pour cela, le biologiste pourra s'aider de la liste minimale d'analyses toxicologiques d'urgence recommandée par la Société Française de Toxicologie Analytique (10) pour définir une liste propre à l'établissement qui tiendra compte de ses spécificités et des priorités retenues par les services cliniques locaux.

La collaboration clinico-biologique ne se limite pas à un dialogue en amont de la démarche toxicologique, mais comprend aussi une discussion des résultats qui permet au clinicien et à l'analyste d'apporter leur compétence dans leur interprétation et de décider soit de l'arrêt des investigations soit le cas échéant d'une recherche complémentaire ou d'une surveillance de la concentration du toxique (toxicocinétique).

\section{Méthodes analytiques de dépistage et/ou de confirmation}

Pour répondre à cette demande toxicologique, le biologiste dispose de nombreuses méthodologies dont les caractéristiques en termes de spécificité, sensibilité, rapidité et facilité de mise en œuvre sont très différentes $(11,12,13)$. L'hétérogénéité des laboratoires ne devant pas être un obstacle à une conduite analytique de qualité, il convient d'identifier toutes ces méthodes avec leurs avantages et leurs limites pour les intégrer dans une stratégie locale performante.

A côté des méthodes séparatives, qui jouent un rôle de plus en plus important en toxicologie d'urgence et des techniques immunologiques au domaine d'application limité mais très diffusées ces dernières années, il existe également des méthodes colorimétriques moins spécifiques et des méthodes enzymatiques permettant une première réponse rapide face à une intoxication aiguë.

\section{Méthodes colorimétriques, photométriques et enzymatiques}

Ce groupe de méthodologies se place dans un contexte d'urgence et ne nécessite pas d'investissement spécifique en matériel. Ce sont des techniques adaptables à toute structure, quelle que soit leur taille, et compatible avec l'urgence toxicologique si l'on en connait les limites et si elles s'intègrent dans une démarche complémentaire associant sur le site ou en collaboration avec un centre de proximité d'autres méthodes plus performantes et plus spécifiques.

Parmi ce groupe, il est intéressant de citer la mise en évidence dans les urines ou le liquide gastrique, par colorimétrie, des molécules ou famille de molécules comme les carbamates (14) (réaction au furfural) ou le paraquat (réaction à la dithionite).

Cette approche non spécifique, dépendante des risques d'interférences peut cependant se justifier comme une première étape d'orientation en confirmant ou infirmant la présence de toxiques présumés lors de la découverte du patient. Les techniques photométriques d'émission sont utilisées essentiellement pour la détermination de la concentration en lithium lors d'intoxications chez des patients traités au long cours. Elles permettent le dosage et le suivi thérapeutique pour un recours éventuel à une épuration extra-rénale. De même la mesure spectrophotométrique de la carboxyhémoglobinémie permet de répondre en urgence à une intoxication par le monoxyde de carbone.

En biologie clinique, de très nombreux dosages font appel à une réaction enzymatique dont la maîtrise méthodologique existe dans tous les laboratoires d'où son interrêt potentiel en toxicologie d'urgence. Ainsi, un certain nombre de méthodes enzymatiques ont été proposées dans l'intention de rendre plus aisée l'utilisation et donc la diffusion de ces dosages. C'est le cas tout particulièrement pour l'éthanol (15), le méthanol, l'isopropanol, l'éthylène glycol ou pour certains métabolites comme l'acide formique, glycolique ou oxalique (16). En effet, les alcools et glycols se prêtent bien à des réactions d'oxydation avec formation d'un aldéhyde ou d'une cétone sous l'action d'enzymes telles que les déshydrogénases comme l'alcool déshydrogénase (ADH) ou les oxydases.

L'enzymologie peut également être utilisée dans la mise en évidence d'une intoxication cyanhydrique par la mesure de la lactacidémie. De même, le dosage de 
cholinestérase plasmatique comme indicateur biologique indirect de toxicité des organophosphorés permet une réponse en urgence.

En comparaison des méthodes physiques (CPG, CLHP, $\mathrm{EC}, \mathrm{RMN}, \ldots$ ) les techniques enzymatiques offrent plusieurs avantages tels qu'un appareillage et un savoir-faire présent dans tous les laboratoires ainsi qu'un délai d'obtention plus court, d'où leur bonne adaptation à l'urgence. Cependant, le principal défaut est leur manque de spécificité. Les interférences observées doivent être replacées dans le contexte clinique auquel sont destinées ces méthodes.

\section{Méthodes immunologiques}

L'immunoanalyse par compétition réalisée en phase liquide ou hétérogène est une méthode facile à mettre en œuvre, automatisable et rapide (17). De nouveaux tests immunologiques, à usage unique sur support solide, dédiés au dépistage urinaire complètent cette offre : leur interprétation nécessite de connaître les limites de ce type d'analyse.

L'immunoanalyse permet la mise en évidence, à l'aide d'un anticorps de classe à spécificité large, de grandes familles de médicaments (benzodiazépines, barbituriques, antidépresseurs tricycliques) et de substances illicites (cannabinoïdes, opiacés, cocaïne, amphétamines, ...). Elle s'applique également au dosage plasmatique de médicaments à risque toxique (carbamazépine, phénytoïne, valproate, digoxine, paracétamol, salicylés, théophylline, méthotrexate, ... ), mais, dans ce type d'analyse, l'anticorps est à spécificité étroite.

Les partenaires de la réaction immunologique, l'antigène traceur et l'anticorps, jouent un rôle essentiel qu'il faut bien connaître pour interpréter correctement le résultat d'une immunoanalyse. La nature de l'antigène ainsi que la concentration retenue pour établir un seuil de positivité ont un rôle clé puisque chaque résultat sera interprété en comparant la réponse de cette molécule à ce seuil. La diversité des réponses des différentes molécules d'une même classe vis-à-vis d'un anticorps doit rendre le biologiste et le clinicien prudents dans leur interprétation des résultats d'un test immunologique. Citons pour exemple la reconnaissance des différentes benzodiazépines (18) dont l'interprétation tiendra compte du choix de l'antigène retenu par le fournisseur (oxazépam ou nordiazépam), du seuil de positivité choisi (100 à $300 \mu \mathrm{g} / \mathrm{l})$, de la réactivité des différentes benzodiazépines et, selon la matrice, de la présence de la molécule mère et/ou de ses métabolites. L'utilisation de trousses immunologiques pour le dépistage des antidépresseurs pose également de nombreux problèmes (19). Bien adaptées à la mise en évidence des antidépresseurs imipraminiques tricycliques, elles sont inaptes à la détection des antidépresseurs tétracycliques, des IMAO et des antidépresseurs non IMAO non tricycliques (ISRS, IRSNA,...) dont la prescription médicale est de plus en plus fréquente (20). Il est donc indispensable de bien connaître la réactivité croisée de toutes les molécules d'une même famille.

L'exemple des réactifs immunologiques pour la recherche urinaire des opiacés et des amphétamines est tout à fait significatif des erreurs graves d'interprétation qu'il faut absolument éviter. Le métabolisme des opiacés conduit à l'élimination urinaire de la morphine libre ou conjuguée. C'est en réalité le noyau morphinane qui est reconnu par l'anticorps des trousses commerciales, et les urines contenant des molécules à usage thérapeutique : codéine, codéthyline, pholcodine ou des molécules d'usage illicite, 6-monoacétylmorphine (métabolite de l'héroïne) seront indifféremment reconnues dès que leur concentration sera supérieure au seuil de positivité du test. A l'inverse aucun des morphinomimétiques (buprénorphine, méthadone, dextropropoxyphène, ...), dont la structure chimique correspond à un noyau morphinane modifié, n'entraînera de positivité, même pour à usage abusif.

L'utilisation des trousses "amphétamines" (anticorps polyclonaux ou monoclonaux) pour la recherche d'ecstasy demande la même prudence d'interprétation (21) : les analogues structuraux à usage thérapeutique courant, comme les décongestionnants des voies nasales (éphédrine, phényléphrine,...) ou comme les anoréxigènes (clobenzorex, fenfluramine,...) seront aussi détectés. Il est donc impératif de confirmer la positivité de ces deux "familles", opiacés et amphétamines, par la complémentarité d'une méthode chromatographique. Par ailleurs, il ne faut pas oublier que les méthodes de dépistage immunologiques sont sensibles à de nombreuses substances adultérantes utilisées par les toxicomanes pour masquer la détection des substances illicites (22). Certains de ces adultérants (variation de $\mathrm{pH}$, glutaraldéhyde, créatinine, nitrites, ...) sont identifiables à l'aide de bandelettes réactives.

\section{Méthodes séparatives}

Les techniques de séparation utilisées en toxicologie pour détecter, identifier et doser une molécule toxique, regroupent principalement les méthodes chromatographiques et plus récemment électrophorétiques avec l'électrophorèse capillaire et ses variantes (23). Depuis quelques années, ces méthodes prennent une part de plus en plus importante dans le screening toxicologique car elles permettent de caractériser un éventail très large de molécules. (24). 
Les méthodes chromatographiques se fondent sur le coefficient de partage des substances à analyser entre deux phases, dont l'une est immobile (phase stationnaire) et l'autre mobile.

Elles permettent l'analyse des liquides biologiques, quelle qu'en soit la nature, après une étape d'extraction (liquide/liquide ou liquide/solide) des médicaments et xénobiotiques rendue nécessaire du fait de la complexité du milieu biologique.

Les substances séparées sont analysées en sortie de colonne par un détecteur approprié à la molécule recherchée et à la technique utilisée. La CLHP peut être couplée à la spectrométrie UV (réseau oscillant, barrettes de diodes), la fluorimétrie, l'ampérométrie, la réfractométrie ou la spectrométrie de masse alors que les détecteurs les plus utilisés en CPG sont du type FID, NPD, capture d'électrons et spectromètre de masse

Parmi les molécules inscrites sur la liste minimale des examens toxicologiques, à effectuer en urgence, les méthodes chromatographiques permettent de doser certaines substances à risque toxique parmi lesquelles le méprobamate par CPG-FID (25). Cependant, dans le cadre de la toxicologie d'urgence, l'intérêt des méthodes séparatives est de permettre une recherche aussi large que possible afin d'identifier et de doser le maximum de molécules. Cette démarche de screening peut être automatisée ou manuelle et s'accompagne d'une étape d'extraction nécessaire pour isoler les toxiques des autres constituants présents dans le liquide biologique. Cette étape peut être réalisée au sein d'un automate (extraction en ligne, Remedi ${ }^{\oplus}$, ) ou manuelle en phase liquide ou en phase solide. L'extraction classique liquide/liquide se résume à un partage de la substance entre deux solvants. Elle est d'autant plus performante que sa solubilité est forte dans un solvant et faible dans l'autre. Des améliorations de cette extraction sont possibles (ultrasons, micro-ondes, solvants ternaires). L'extraction liquide/solide grâce à de nouveaux supports d'extraction $\left(\right.$ Oasis $^{\circledR}$, Bond Elut Certify ${ }^{\oplus}$, Isolute ${ }^{\oplus}, \ldots$ ) peut être semi automatisable et facilement standardisable.

L'utilisation de la CPG en screening toxicologique est très répandue et standardisée grâce à sa facilité de couplage avec la spectrométrie de masse qui permet l'identification des molécules. Elle représente encore actuellement la méthode de choix pour l'identification d'un toxique non identifié lors d'un screening préliminaire. Cependant, même si son utilisation est rendue de plus en plus aisée par l'utilisation de l'informatique, elle nécessite encore un personnel spécialisé et un budget d'investissement non négligeable. De plus, la thermolabilité et/ou la faible volatilité de certaines molécules nécessitent souvent une étape supplémentaire de dérivatisation.
A côté de cette technique de référence, la chromatographie sur couche mince a beaucoup progressé par une meilleure compréhension des phénomènes mis en jeu dans la migration. Elle offre une grande souplesse, mais le prix des appareils freine son développement. Récemment, la chromatographie couplée à l'enregistrement des spectres UV est venue compléter l'approche toxicologique. L'enregistrement de l'ensemble du spectre UV par des spectrophotomètres à barrettes de diodes ou à réseau oscillant couplé à une séparation par chromatographie liquide haute performance (CLHP) apporte une solution satisfaisante, à la fois par une utilisation plus accessible que la détection par spectrométrie de masse type CG/SM ou LC/SM et un investissement moins important.

Les techniques d'identification en chromatographie liquide couplée à l'enregistrement des spectres UV se répartissent en deux groupes :

- Les méthodes développées au sein du laboratoire

Ces systèmes modulaires, dont les conditions analytiques préalablement définies par chaque utilisateur pour le screening toxicologique, sont spécifiques à chaque site et nécessitent la réalisation d'une bibliothèque personnelle à partir d'un algorithme d'identification non standardisé (26)

- Les méthodes proposées par les fournisseurs sous la forme soit :

- d'un concept ouvert autour d'une chaîne CLHP avec des conditions analytiques fixées, une bibliothèque informatique et un algorithme imposé (AllianceMillenium $^{\oplus}$, Waters) (27).

- d'un concept fermé automatisé comprenant la préparation et la purification en ligne de l'échantillon, la séparation chromatographique et l'exploitation informatique selon un algorithme d'identification à partir d'une base de données transposable (Remedi ${ }^{\oplus}$, Biorad) $(28,29)$.

\section{Mise en place d'une démarche analytique en toxicologie d'urgence Protocole services cliniques-biologie}

L'optimisation de l'approche biologique et du diagnostic toxicologique nécessite l'élaboration d'une stratégie analytique, définie localement avec les services cliniques concernés, sur un certain nombre de points indispensables à la prise en charge de l'intoxication aiguë. Ainsi, il est important :

- d'obtenir un consensus sur une liste minimale d'analyses toxicologiques à effectuer en urgence. 
- d'identifier clairement la procédure retenue selon l'heure d'arrivée du prélèvement (en particulier pour les heures ouvrables et celles de garde du laboratoire). - de bien définir le délai de réponse souhaité et d'afficher clairement la durée d'analyse nécessaire à sa réalisation.

Tous ces éléments sont indispensables à une bonne coordination clinico-biologique, car ils prennent en compte les spécificités locales telles que l'équipement existant au laboratoire, la formation et disponibilité du personnel et le cas échéant la collaboration avec un centre spécialisé de proximité. Cette transparence est le gage d'un bon fonctionnement et permettra d'éviter des quiproquos quant à l'interprétation des résultats fournis par le laboratoire suite à un malentendu lié aux besoins de l'un et aux potentialités analytiques de l'autre.

\section{Le prélèvement}

Pour détecter les toxiques dans les liquides biologiques, le laboratoire doit tout d'abord disposer d'échantillons adéquats. Pour cela, quelques règles importantes doivent être respectées :

- Absence de toute contamination de l'échantillon lors du prélèvement par les solutions désinfectantes (alcool, produits iodés,..) ou autres (gel à la lidocaïne, ...).

- Choix des tubes spécifiques à certaines analyses

- Fermeture hermétique des tubes pour prévenir l'évaporation des substances volatiles et la contamination par les micro-organismes.

- Collecte suffisante d'échantillons à l'admission afin que des analyses rétrospectives restent possible si l'évolution clinique le rend nécessaire (sérothèque, urothèque, ...)

Ces items respectés, il convient de prélever les échantillons biologiques adéquats. Ainsi, en toxicologie d'urgence, le sang doit être considéré comme un milieu biologique privilégié. La concentration sanguine des toxiques est en effet le plus souvent corrélée soit à un facteur de gravité, soit à un facteur pronostic. De plus, le prélèvement sanguin lors d'une admission ne pose généralement pas de problèmes. Le prélèvement urinaire reste cependant indispensable pour le dépistage des stupéfiants et des substances difficiles à doser dans le sang (paraquat, diquat, ...) ou facilement identifiables dans l'urine (carbamates, ...). A titre complémentaire de l'analyse sanguine, les urines peuvent être utilisées pour la confirmation du ou des toxiques ingérés et l'identification de ces métabolites ce qui permet d'établir une première chronologie de l'intoxication.

L'échantillon de liquide gastrique peut être très utile lorsque l'histoire clinique suggère une ingestion récen- te car il contient de grande quantité de toxique non métabolisé. Son utilisation est de moins en moins fréquente du fait de son abandon progressif et de l'instauration d'un traitement au charbon.

D'autres types d'échantillons impliqués dans l'intoxication peuvent aussi être analysés (LCR, comprimés, sachets, ....). Il faut cependant garder à l'esprit que seule la mise en évidence du toxique dans un liquide biologique prouve son implication dans le tableau clinique du patient.

\section{Démarche analytique}

La stratégie analytique mise en place dépendra de l'équipement présent au sein du laboratoire.

\section{Dépistage toxicologique}

En l'absence de méthodes séparatives, la démarche consiste à utiliser dans un premier temps des techniques qualitatives souvent peu spécifiques qui vont fournir en revanche des résultats présomptifs rapides et qui seront secondairement confirmés par des méthodes plus sophistiquées et plus spécifiques. Le principe du dépistage se définit comme une recherche rapide dans les milieux biologiques des toxiques les plus souvent impliqués dans l'épidémiologie locale. Il consiste ainsi à rechercher ou à exclure de manière systématique les toxiques par des méthodes analytiques simples et rapides. Ce type de dépistage tente de détecter une série de substances ciblées dans les trois milieux biologiques principaux : sang, urines et liquide gastrique et peut être envisagé en fonction des circonstances et de la présentation clinique (toxicomanie, ingestion médicamenteuse, ...)

Cette première étape toxicologique fait appel à l'immunoanalyse et aux méthodes colorimétriques ou enzymatiques. Cette analyse qualitative dont les limites doivent être clairement identifiées et exprimées n'a pour objectif que de confirmer la présomption du clinicien. Elle possède cependant l'avantage de pouvoir être mise en œuvre par tous les laboratoires d'analyses médicales. Elle ne nécessite pas de traitement préanalytique, mais reste limitée car elle ne permet pas de mettre en évidence de nombreux produits responsables d'intoxications graves. De plus, tout résultat positif devra être confirmé par une méthode plus spécifique.

\section{Screening toxicologique}

Le progrès récent des méthodes séparatives se traduit par une utilisation plus aisée grâce à l'apport de l'informatique et à un coût d'investissement qui s'est considérablement réduit. Ce constat permet de comprendre et d'encourager le développement de ces techniques dans le cadre de l'urgence. En effet, ces méthodes essentiellement chromatographiques sont capables de détecter et 
d'identifier un nombre important de xénobiotiques avec une grande sélectivité. Elles font appel en général à la chromatographie sur couche mince, à la chromatographie liquide haute performance couplée à un détecteur de type barrettes de diodes ou balayage UV, automatique ou non, et à la chromatographie en phase gazeuse couplée à un spectromètre de masse.

Après l'identification d'un toxique et chaque fois que cela sera nécessaire, la confirmation par une autre méthode sera quantitative afin de mieux documenter l'intoxication. Elle s'avère nécessaire pour la poursuite de traitements coûteux ou invasifs comme l'instauration :

- d'une hémodialyse (lithium, méthanol, phénobarbital) ou d'une hémoperfusion (méprobamate, phénobarbital, théophylline)

- d'un traitement antidotique (paracétamol, méthanol, éthylène glycol, digitaliques)

- d'une diurèse forcée ou alcaline (salicylés, méprobamate)

L'approche analytique en toxicologie ne peut se réduire à une seule technique même séparative. Il est donc nécessaire de bâtir un algorithme suivant son équipement et la nature du prélèvement en tenant compte des priorités préalablement établies avec les services cliniques. Un exemple est présenté dans le tableau I pour un site ayant retenu la liste minimale d'analyses toxicologiques d'urgence recommandée par la SFTA et disposant de méthodes séparatives compatibles avec une utilisation $24 \mathrm{~h} / 24$.

\section{Conclusion}

L'analyse toxicologique dans le cadre des urgences hospitalières est un complément de l'approche clinique et présente des exigences spécifiques : facilité de mise en œuvre, obtention rapide de résultats, couverture d'un large éventail de xénobiotiques et disponibilité. Pour réaliser cette activité, il n'existe pas une technique idéale, d'où la nécessité de choisir plusieurs méthodes complémentaires en fonction de la stratégie adoptée dans l'établissement. Le choix de l'analyste doit privilégier les méthodes séparatives, mais il dépend surtout de la vocation de son laboratoire, des priorités définies par les services cliniques locaux, des contraintes techniques et économiques en matière d'équipement, du personnel et de la proximité de laboratoires plus spécialisés.

Les recherches toxicologiques ne supplantent jamais l'expérience clinique dans le diagnostic et l'évaluation des intoxications aiguës. Cependant, la capacité des analyses à accroître la certitude diagnostique ne fait pas de doutes, même si l'utilité des investigations toxicologiques est parfois controversée du fait de l'absence d'une collaboration multidisciplinaire.

Le dialogue et la coopération entre le clinicien et l'analyste sont d'importance primordiale et représentent la meilleure garantie pour assurer une utilisation optimale du laboratoire et l'interprétation multidisciplinaire des résultats en tenant compte de la toxicocinétique des substances impliquées et des multiples causes possibles de discordance entre la clinique et les données analytiques.

\section{Démarche analytique en Toxicologie d'Urgence}

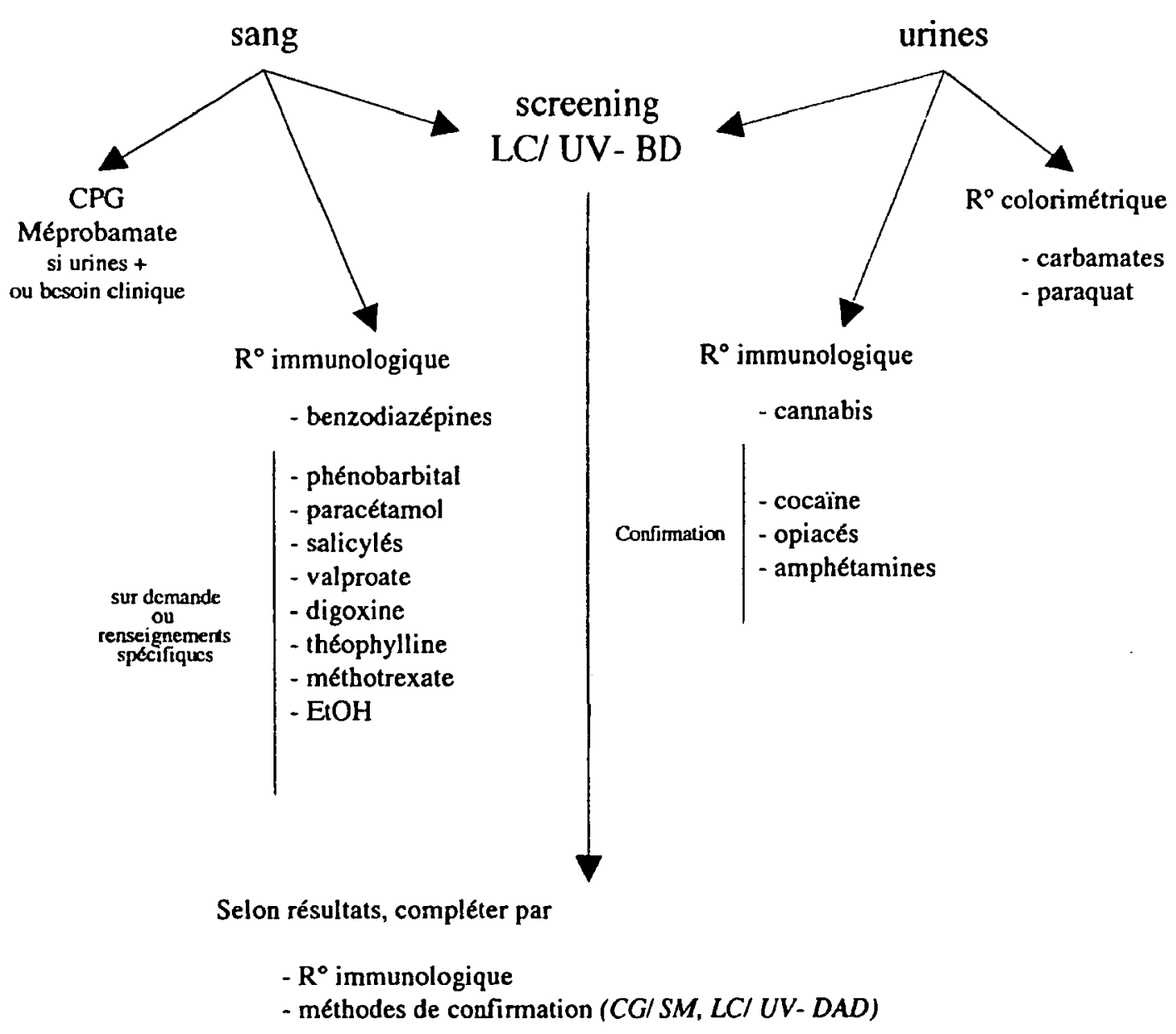




\section{Références}

1. Savuyc P., Hanna J., Danel V. Epidémiologie des intoxications : Plus de 2000 décès par an. Rev. Prat., 1999 ; 481 : 2054-57.

2. Baud F.J. L'analyse toxicologique en urgence. JEUR. $1989 ; 2: 70-2$.

3. Lheureux P., Askenasi R., Maes V. Du bon usage du laboratoire de toxicologie. 2e partie : Utilité clinique et interprétation de résultats. Réan. Urg., 1996 ; 3 : 341-52.

4. Lheureux P., Askenasi R., Maes V. Du bon usage du laboratoire de toxicologie. 1e partie : aspects techniques. Rean. Urg., 1996 ; 2 : 87-95.

5. Lheureux P., Garbusinski J., Devuyst F., D'Eugenio S. Apport de la biologie et de la clinique dans l'évaluation des intoxications aiguës, Rev. Fr. Lab., 1999 ; 312 : 35-41.

6. Jaeger A., Mangin P. L'analyse toxicologique en urgence, intérêts et limites. Rev. Prat. 1994 ; 5 : 287-92.

7. Laforge M., Levillain P., Gourlain H., Buneaux F., Galliot M. Analyses biologiques : critères indirects d'une intoxication aiguë. Rev. Fr. Lab., 1999 ; 312 : 42-5.

8. Bismuth C., Baud F.J., Conso F., Frejaville J.P., Garnier R., Jeager A. Toxicologie clinique, 5e ed. Flammarion, 1995.

9. Baud F.J. Pharmacokinetic-pharmacodynamic relationships. How are they useful in human toxicology? Toxicol. Lett., $1998 ; 102-103: 643-8$

10. Capolaghi B., Charlier C., Feuillu A.. Gruson A., Houdret N., Kergueris M.F., Lachatre G., Lacroix C., Lamiable D., Plomteux G. Toxiques et analyses biologiques en toxicologie hospitalière. Toxicorama, 1998 ; $3: 128-31$.

11. Houdret N., Lhermitte M. Toxicologie hospitalière : les différentes réponses. Opt. Bio., 1997 ; 192 : 23-8.

12. Pechard A., Besson A., Mialon A., Berny C., Manchon M. Analyse critique des différentes méthodes utilisées pour le dépistage toxicologique dans un laboratoire d'urgence. Ann. Biol. Clin., 1999 ; 57 : 524-37.

13. Lafargue $P$. Aspects généraux de la toxicologie analytique hospitalière. Rev. Fr. Lab., 1996 ; 282 : 95-6.

14. Kergueris M.F., Thomas A., Bourin M. Recherche du méprobamate en toxicologie d'urgence : identification dans les urines. Ann. Biol. Clin., 1994 ; 52 : 469-70.

15. Malandain H., Bourdon J.H., Cano Y., Capolaghi B., David P., Lachatre G., Lacroix C., Lamiable D., Lardet G., Lenormand I., Levillain P., Pollet J., Verchain S., Vincent F. Étude multicentrique du dosage automatisé de l'éthanolémie par méthode enzymatique. Toxicorama, $1997 ; 3$ : 157-69.

16. Malandain H., Cano Y. Place des techniques enzymatiques dans le diagnostic et le suivi des intoxications par un alcool ou un glycol. In. : SFTA Ed. Alcools et Glycols, 1999 ; 36-53.
17. Chinelato $M$. Intérêt des dosages toxicologiques de dépistage en toxicologie d'urgence. A propos de 690 cas de tentatives de suicides médicamenteuses. Th. D. Med., 1996 ; Lyon.

18. Moulsma M., Lardet G., Pascal P., Gagnieu M.C., Gaulier J.M., Vallani J.J. Dépistage immunologique des benzodiazépines dans le sang et les urines. Toxicorama, $1996 ; 3: 23-8$.

19. Goulle J.P., Lacroix C., Nouveau J., Rigaud J.P. Intoxication par les antidépresseurs tricyliques, apport du laboratoire dans la prise en charge médicale. Toxicorama, $1996 ; 4: 21-5$.

20. Marty H., Hoizey G., Lamiable. D., Vistelle R. Intoxications aiguës par les antidépresseurs. Rev. Fr. Lab. $2000 ; 322: 53-9$.

21. Verstaete A., Van Haue I. Spécificité des immunoessais pour la détection des amphétamines. Toxicorama, 1997 ; $2: 65-71$.

22. Dumestre-Toulet V. Verstraete A. Les adultérants des tests urinaires. Toxicorama, 1999 ; 3 : 155-66.

23. Labat L., Deveaux M., Dubost J.P. Applications de l'électrophorèse capillaire en toxicologie clinique et médico-légale. Ann. Tox. Anal. 2000 ; 3 : 179-95.

24. Levillain P. Place des méthodes de séparation dans le screening toxicologique d'urgence. Toxicorama, 1998 ; $3: 132-35$.

25. Lamiable D., Kergueris M.F., Capolaghi B., Houdret N. Feuillu A., Lachatre G., Lacroix C., Vincent F., Lardet G., Arditi J., Mura P., Furet Y. Le méprobamate : étude multicentrique et recommandantions. Toxicorama, 1998 ; $3: 136-40$.

26. Capolaghi B., Lacroix C. Screening toxicologique comparé sur le système Remedi et CLHP-BD. Toxicorama, $1998 ; 3: 141-46$.

27. Houdret N., Vinner E., Courtois P., Mathieu D., Lhermitte M. Les méthodes physiques en toxicologie hospitalière. Apports et limites de la chromatographie liquide dans le dépistage des intoxications aiguës : comparaison de la CLHP-Remedi et CLHP-BD Alliance. Toxicorama, $1998 ; 3: 147-52$.

28. Capolaghi B. Utilisation d'un automate de chromatograhie liquide en toxicologie d'urgence. Rev. Fr. Lab., 1996 ; $282: 185-86$.

29. Sadeg N., Dumontet M. Place du Remedi, dans un screening toxicologique. Expérience de 12 mois dans un hôpital général. Toxicorama, $1999 ; 3: 183-7$. 